\title{
QUANTOS EXAMES SÃO NECESSÁRIOS PARA ADQUIRIR APTIDÃO EM COLONOSCOPIA?
}

\author{
Fernanda Prata Borges Martins THULER, Veruska Oliveira Di SENA, \\ Cristina Marfinatti HERVOSO, Ermelindo Della LIBERA e Angelo Paulo FERRARI
}

RESUMO - Racional - Capacitação para realização de colonoscopia implica em competência técnica e cognitiva. A Sociedade Americana de Endoscopia sugere que 100 colonoscopias supervisionadas sejam necessárias para tal. Não há recomendações específicas no Brasil. Objetivo - Avaliar o desempenho e progresso técnico de médicos durante programa de treinamento em colonoscopia. Materiais e Método - Dois residentes em gastroenterologia da Universidade Federal de São Paulo, SP, foram acompanhados prospectivamente no seu primeiro ano de treinamento em colonoscopia. Os parâmetros avaliados foram: capacidade de atingir o ceco, tempo de chegada ao ceco, duração total do exame, identificação das lesões e desconforto do paciente. Resultados - Foram realizadas 271 colonoscopias pelos dois residentes (186 pelo residente A e 85 pelo B). Foram excluídos 27 casos por lesões obstrutivas ou antecedente de cirurgia do cólon, restando 171 e 72 exames, respectivamente, para avaliação dos parâmetros propostos. A taxa de sucesso em alcançar o ceco sem ajuda foi $82,5 \%$ e $56,9 \%$, respectivamente, para A e B. Nos primeiros 72 exames foi: $72,2 \%$ (A) e 56,9\% (B), diferença estatisticamente significativa. O residente A atingiu o ceco em $76 \%$ dos primeiros 100 exames e após o $101^{\circ}$, a taxa aumentou para $91,5 \%$. O tempo médio de chegada ao ceco foi 17,7 e 23,5 minutos para os residentes A e B. Para o examinador A o tempo foi de 19,8 e 14,7 minutos antes e depois do $100^{\circ}$ exame. Discussão - Observou-se que o sucesso em atingir o ceco e o tempo necessário para alcançá-lo melhoraram proporcionalmente ao número de exames realizados, com significância estatística. Contudo, diferenças individuais sugerem que, talvez, 100 colonoscopias seja número insuficiente para aquisição da competência técnica. Conclusão - Apesar de avaliar a curva de aprendizado de apenas dois residentes, observou-se melhora estatisticamente significativa na taxa e tempo de chegada ao ceco com o número de exames realizados. Dependendo, porém, de aptidões individuais, talvez mais de 100 exames sejam necessários no treinamento.

DESCRITORES - Colonoscopia. Competência clínica.

\section{INTRODUÇÃO}

A capacitação para realização da colonoscopia implica na associação da habilidade de manipular adequadamente o aparelho e seus acessórios (competência técnica), à capacidade de identificar e interpretar os achados endoscópicos, e planejar terapêutica efetiva e apropriada (competência cognitiva). A certificação de competência em colonoscopia é geralmente baseada no número de procedimentos supervisionados e na avaliação subjetiva dos instrutores ${ }^{(1,3,6,7,9)}$.

A Sociedade Americana de Endoscopia (ASGE) $)^{(5,10)}$ sugere que 100 colonoscopias supervisionadas seja o número mínimo necessário para que a competência técnica e cognitiva sejam alcançadas. Contudo, existem poucos dados na literatura que investiguem quais parâmetros no treinamento asseguram como a competência é adquirida ${ }^{(4,8)}$.

\section{Objetivo}

Avaliar o desempenho e progresso técnico de médicos residentes em gastroenterologia durante programa de treinamento em colonoscopia. Avaliar quantos exames realizados sob supervisão são necessários para aquisição da capacitação técnica e cognitiva inicial.

\section{MATERIAIS E MÉTODO}

Dois médicos do quarto ano de residência em gastroenterologia da Universidade Federal de São Paulo foram acompanhados por instrutor durante seu primeiro ano de treinamento e aprendizado em colonoscopia, entre fevereiro de 2003 e janeiro de 2004. Os exames foram realizados consecutivamente neste período, sempre iniciados por um deles sob supervisão de um instrutor do setor. Durante o procedimento, o preceptor orientava normalmente o médico residente sem, porém, identificar qualquer alteração até que o mesmo expressasse sua própria opinião. $\mathrm{O}$ instrutor assumiu o exame quando o residente não conseguiu avançar o colonoscópio ou quando julgou necessário, levando em consideração a segurança e o conforto do paciente.

Todas as colonoscopias foram realizados com videocolonoscópios. Os parâmetros avaliados foram: capacidade de atingir o ceco, tempo de chegada ao ceco, duração total do exame, intubação ileal e identificação das lesões.

Disciplina de Gastroenterologia da Universidade Federal de São Paulo - UNIFESP, São Paulo, SP

Endereço para correspondência: Dr. Angelo Paulo Ferrari - Rua Machado Bittencourt, 379 apt. 91 - 04044-001 - São Paulo, SP. E-mail: angelo@gastro.epm.br 
A contagem do tempo tinha início no momento em que o aparelho era introduzido pelo ânus, verificada quando o ceco era alcançado e interrompida ao término do exame. A chegada no ceco foi confirmada pela identificação da válvula ileocecal, apêndice cecal e, quando possível, pela intubação do íleo terminal.

A taxa de sucesso em atingir o ceco foi calculada com base em todos os exames completados pelo residente em treinamento ou pelo preceptor. Pacientes com antecedentes de ressecção colônica, massa obstrutivas ou colostomias foram excluídos do cálculo da taxa de sucesso de chegada no ceco.

A sedação utilizada foi midazolam, meperidina, diazepam e propofol, em doses individualizadas, associados ou não.

A análise estatística foi realizada através do teste de KruskallWallis e teste de comparação de proporções com o programa Primer of Biostatistics $^{\circledR}$. O resultado foi considerado estatisticamente significativo quando valor de $P$ encontrado foi $<0,05$.

\section{RESULTADOS}

No período do estudo foram realizadas 271 colonoscopias pelos dois médicos residentes. O residente A realizou 186 exames e o B os demais 85 . Foram excluídos 27 casos devido à presença de lesões obstrutivas ou antecedente de cirurgia do cólon (15 e 13), restando então 171 e 72 exames, respectivamente.

Quanto à distribuição por sexo e idade, no grupo do residente $\mathrm{A}$ havia 107 mulheres, com média de idade de 55,4 $( \pm 14,7)$ anos e no grupo B 42 mulheres, com média de idade de $55( \pm 15,6)$ anos.

A taxa de sucesso em alcançar o ceco sem auxílio do preceptor foi de $82,5 \%$ (141/171 exames) e 56,9\% (41/72 exames), respectivamente para A e B (Tabela 1). Avaliando este índice para os primeiros 72 exames de ambos, observou-se que o residente A atingiu o ceco em $72,2 \%$, enquanto o B em 56,9\% das vezes, $P=0,001$ (Tabela 2). A Tabela 1 mostra que a taxa de sucesso foi de $76 \%$ nos primeiros 100 exames e de $91,5 \%$, a partir de então, para o residente A $(P=0,015)$.

TABELA 1 - Taxa de sucesso e tempo de chegada ao ceco para os residentes $\mathrm{A}$ e $\mathrm{B}$

\begin{tabular}{lcc}
\hline & $\begin{array}{c}\text { Taxa de chegada } \\
\text { ao ceco }(\%)\end{array}$ & $\begin{array}{c}\text { Tempo de chegada } \\
\text { ao ceco (minutos) }\end{array}$ \\
\hline Residente A (171 exames) & 82,5 & 17,7 \\
Residente A (até $100^{\circ}$ exame) & $76^{*}$ & $19,8^{* *}$ \\
Residente A (após $101^{\circ}$ exame) & $91,5^{*}$ & $14,7 * *$ \\
Residente B (72 exames) & 56,9 & 23,5 \\
\hline$* P=0,015$ & & \\
$* * P=0,001$ & &
\end{tabular}

TABELA 2 - Taxa de sucesso de chegada ao ceco para os residentes A e B nos primeiros 72 exames

\begin{tabular}{lc}
\hline & Taxa de chegada ao ceco (\%) \\
\hline Residente A (até $72^{\circ}$ exame) & 72,2 \\
Residente B & 56,9 \\
\hline$P=0,001$ &
\end{tabular}

A intubação do íleo terminal foi bem sucedida em 68,4\% (A) e 36,1\% (B) das colonoscopias, sendo que para o examinador A este índice foi de $62 \%$ e $77,5 \%$, respectivamente até o $100^{\circ}$ exame e após $(P=0,06)$.

O tempo médio de chegada até o ceco em todos os exames foi 17,7 e 23,5 minutos, respectivamente para os residentes A e B. A análise dos dados dos primeiros 72 exames não revelou diferença estatística.
O tempo médio necessário para o examinador A atingir o ceco nos primeiros 100 exames foi de 19,8 minutos, enquanto a partir do $101^{\circ}$ exame esta média caiu para 14,7 minutos $(P=0,001)$, como listado na Tabela 1. Avaliou-se, também, a evolução do tempo necessário para que o ceco fosse alcançado ao longo dos exames em uma curva de tendência (Gráfico 1). A média da duração total do exame foi 39,5 e 50,2 minutos, respectivamente para A e B.

GRÁFICO 1 - Curva de tendência da evolução do tempo de chegada ao ceco pelos residentes A e B

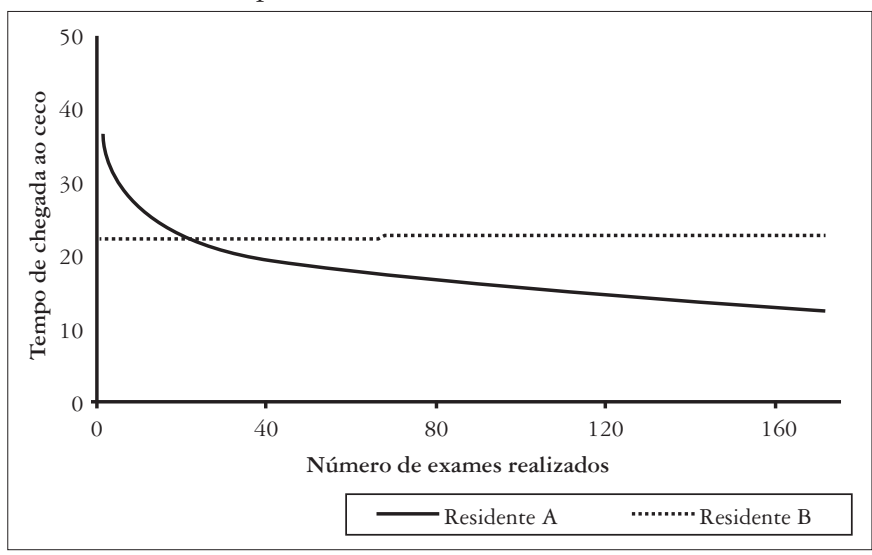

Os pontos anatômicos atingidos quando a colonoscopia não pôde ser completada pelo residente estão listados na Tabela 3. Nesta situação o exame foi assumido pelo preceptor, que atingiu com sucesso o ceco em $99,6 \%$ das vezes, não completando o exame em apenas um caso.

TABELA 3 - Ponto anatômico alcançado em 30 e 31 exames, respectivamente, quando o ceco não pôde ser alcançado pelo médico em treinamento

\begin{tabular}{lll}
\hline Ponto anatômico & Residente A & Residente B \\
\hline Sigmóide & $9(30,0 \%)$ & $9(29,0 \%)$ \\
Descendente & $7(23,3 \%)$ & $12(38,7 \%)$ \\
Transverso & $11(36,7 \%)$ & $8(25,80 \%)$ \\
Ascendente & $3(10,0 \%)$ & $2(6,50 \%)$ \\
\hline
\end{tabular}

Quanto aos diagnósticos, 22,2\% dos exames foram normais. As principais alterações encontradas foram pólipos $(55,1 \%)$, doença diverticular dos cólons $(29,6 \%)$, doença inflamatória intestinal $(6,6 \%)$, alterações vasculares $(4,9 \%)$ e neoplasias $(2,5 \%)$. Os residentes foram capazes de identificar as alterações em $99,5 \%$ (A) e $100 \%$ (B) dos casos, respectivamente. Dentre as complicações, observou-se um caso de sangramento pós-polipectomia $(0,4 \%)$.

\section{DISCUSSÃo}

A indicação da colonoscopia apresentou, recentemente, aumento significativo, principalmente pela introdução de programas de rastreamento e vigilância do câncer colorretal. O objetivo da colonoscopia é a avaliação completa e detalhada de todo o cólon, com desconforto mínimo para o paciente. Vários fatores contribuem para o sucesso do exame: evitar a insuflação excessiva durante a inserção, retirada freqüente para manter o aparelho retificado e uso apropriado de manobras que facilitem a inserção sem a formação de alças ${ }^{(3,8,9)}$. 
A ASGE recomenda que o mínimo de 100 colonoscopias seja realizada pelo profissional em treinamento para aquisição de competência técnica e cognitiva ${ }^{(5,10)}$.

A maioria dos especialistas concorda que para ser considerado experiente, o endoscopista deve ser capaz de atingir o ceco em pelo menos $90 \%$ das vezes ${ }^{(8)}$. Esta taxa deve ser alcançada com treinamento apropriado e experiência.

Poucos estudos foram publicados no sentido de determinar as diretrizes do programa de treinamento em colonoscopia e as definições de critérios para competência foram geralmente baseadas na opinião de especialistas ${ }^{(1,3,6)}$

CASS et al. ${ }^{(1)}$ realizaram estudo prospectivo com 12 médicos residentes, avaliando a aquisição de habilidade técnica durante o treinamento de colonoscopia, através de quatro critérios objetivos: passagem pela flexura esplênica, chegada ao ceco, reconhecimento de uma alteração quando presente, e identificação correta da anormalidade. Observaram sucesso de $80 \%$ após 100 exames, 94\% após 210 e $96 \%$ após 274 , concluindo que mais de 100 colonoscopias supervisionadas são necessárias para alcançar competência técnica.

Em estudo multicêntrico, publicado apenas sob a forma de resumo, CASS et al. ${ }^{(2)}$ sugerem que 140 colonoscopias são necessárias para alcançar índice de sucesso de intubação do ceco de $90 \%$.

No estudo de MARSHALL et al. ${ }^{(6)}$, a freqüência de chegada no ceco em 30 minutos foi avaliada durante os últimos 7 meses de treinamento do primeiro e segundo anos de nove médicos residentes em gastroenterologia e/ou cirurgia. A taxa de sucesso para os residentes do primeiro ano foi de $54 \%(25 \%-86 \%)$, enquanto no segundo ano foi de $86 \%$ (73\%-93\%). O número médio de exames realizados ao final do primeiro ano foi de 149 e 318 ao fim do segundo ano. Concluíram que a capacitação para colonoscopia aumenta com a experiência do profissional e sugerem que o número mínimo de 100 exames recomendado pela ASGE possa ser baixo.

CHURCH et al. ${ }^{(3)}$ acompanharam prospectivamente 18 residentes durante as primeiras 100-125 colonoscopias do programa de treinamento. O exame foi considerado completo quando o ceco foi alcançado, o que ocorreu em $56,4 \%(27,8 \%-83,9 \%)$. Nos primeiros 25 casos, o sucesso foi $43,1 \%$, e após 100 exames, subiu para $75,1 \%$. Esses autores sugerem que os programas de treinamento em colonoscopia devam oferecer pelo menos 150-170 exames.
$\mathrm{O}$ índice de sucesso atingido pelo médico em treinamento no estudo de TASSIOS et al. ${ }^{(8)}$ foi de $67 \%$ (59\%-75\%) e $77 \%$ (66\%-88\%), quando 100 e 180 exames eram realizados, respectivamente, ao fim do segundo e terceiro anos de treinamento. Esses autores concluíram que na dependência de aptidões individuais, entre 100 e 180 exames são necessários para aquisição de competência em colonoscopia.

Em outro estudo ${ }^{(7)}, 334$ colonoscopias consecutivas realizadas por um mesmo médico em treinamento, no período de 3 anos, foram avaliadas. O ceco pôde ser alçando em $67 \%$ dos primeiros 100 exames, $88 \%$ para os 100 seguintes e $95 \%$ para os outros 100 . Não há informação sobre supervisão dos exames e os autores sugerem que 100 a 200 colonoscopias são necessárias para atingir taxa de sucesso de $90 \%$.

No presente trabalho utilizou-se a chegada no ceco como indicador de competência técnica, por ser critério objetivo e de fácil comparação. Os exames realizados em pacientes com lesões obstrutivas ou cirurgia prévia foram excluídos do cálculo da taxa de sucesso. A competência cognitiva foi avaliada pela capacidade do médico reconhecer e interpretar as alterações.

Observou-se que o sucesso e o tempo necessário para atingir o ceco melhoraram proporcionalmente ao número de exames realizados. Quando se analisaram os dados do residente que realizou 171 colonoscopias, encontrou-se diferença estatisticamente significante em ambos os índices antes e após o $100^{\circ}$ exame. Contudo, as diferenças individuais do aprendizado, como a que se encontrou ao comparar a taxa de chegada ao ceco pelos dois residentes mostram que talvez 100 colonoscopias sejam insuficientes para aquisição da competência técnica.

Não há parâmetros da Sociedade Brasileira de Endoscopia Digestiva a respeito do número de exames necessários ou tempo a ser dedicado ao treinamento em colonoscopia.

Apesar de tratar-se de programa de treinamento, a taxa de complicações de $0,4 \%$ foi compatível com dados da literatura ${ }^{(10)}$.

\section{CONCLUSÃO}

Embora este estudo avalie a curva de aprendizado de apenas dois residentes, observou-se que a capacidade de atingir o ceco aumenta de acordo com o número de exames realizados. Dependendo das aptidões individuais, porém, o número mínimo de colonoscopias sugerido pela ASGE pode ser insuficiente para aquisição de competência técnica e também para identificação das lesões.

Thuler FPBM, Di Sena VO, Hervoso CM, Della Libera E, Ferrari AP. How many procedures are necessary to achieve competency in colonoscopy? Arq Gastroenterol 2004;41(4):211-4.

ABSTRACT - Background - Competency for colonoscopy implies technical and cognitive skills. The American Society for Gastrointestinal Endoscopy has suggested 100 supervised procedures might be necessary. There are no specific recommendations in Brazil. Aim - To evaluate technical progress of trainees during a regular colonoscopy training program. Materials and Method - Two gastrointestinal fellows at Federal University of São Paulo, SP, Brazil, were prospectively evaluated during first year training. The frequency and time of reaching the cecum, total procedure duration, ability to identify lesions and patient discomfort were recorded. Results - Two hundred and seventy one colonoscopies were preformed by both fellows (fellow A: 186 and B: 85 ). Twenty-seven cases were excluded of obstructive lesions or previous surgery, leading 171 and 72 exams, respectively. The mean success rate of reaching the cecum was $82.5 \%$ and $56.9 \%$, respectively. For the first 72 exams it was $72.2 \%$ and $56.9 \%$. Fellow A reached the cecum in $76 \%$ of first 100 colonoscopies, improving to $91.5 \%$ after the $101^{\text {st }}$. Mean time for trainees to complete the procedure was 17.7 and 23.5 minutes to fellows A and B. Trainee A took 19.8 and 14.7 minutes before and after the $100^{\text {th }}$ colonoscopy. Discussion - Success rate and time taken to reach the cecum progressively improved over the number of procedures with statistical significance. However, because of individual differences, 100 colonoscopies may be insufficient to acquisition of technical skills. Conclusion - Although analyzing the learning curve of two fellows only, we could notice a statistically significant improve in reaching the cecum with experience over time. However, depending on individual skills more than 100 procedures may be necessary during training.

HEADINGS - Colonoscopy. Clinical competence. 


\section{REFERÊNCIAS BIBLIOGRÁFICAS}

1. Cass OW, Freeman ML, Peine CJ, Zera RT, Onstad GR. Objective evaluation of endoscopy skills during training. Ann Intern Med 1993;118:40-4.

2. Cass OW, Freeman ML, Peine CJ. Surgeons and GI fellows do not differ in the aquisition of endoscopy skills during training [abstract]. Gastrointest Endosc 1994;40:P39.

3. Church J, Oakley J, Milsom J, Strong S, Hull T. Colonoscopy training: the need for patience (patients). ANZ J Surg 2002;72:89-91

4. Dominitz JA, Eisen GM, Baron TH, Goldstein JL, Hirota WK, Jacobson BC, Johanson JF, Leighton JA, Mallery JS, Raddawi HM, Vargo JJ, 2nd, Waring JP, Fanelli RD, Wheeler-Harbough J, Faigel DO. Complications of colonoscopy. Gastrointest Endosc 2003;57:441-5.

5. Guidelines for credentialing and granting privileges for gastrointestinal endoscopy. American Society for Gastrointestinal Endoscopy. Gastrointest Endosc 1998;48:679-82.

6. Marshall JB. Technical proficiency of trainees performing colonoscopy: a learning curve. Gastrointest Endosc 1995;42:287-91.

7. Parry BR, Williams SM. Competency and the colonoscopist: a learning curve. Aust N Z J Surg 1991;61:419-22.
8. Tassios PS, Ladas SD, Grammenos I, Demertzis K, Raptis SA. Acquisition of competence in colonoscopy: the learning curve of trainees. Endoscopy 1999;31:702-6.

9. Teague R, Soehendra N, Carr-Locke D, Segal E, Nagy G, Chao W, Sakai Y. Setting standards for colonoscopic teaching and training. J Gastroenterol Hepatol 2002;17 Suppl:S50-3.

10. Wexner SD, Litwin D, Cohen J, Earle D, Ferzli G, Flaherty J, Graham S, Horgan S, Katz BL, Kavic M, Kilkenny J, Meador J, Price R, Quebbemann B, Reed W, Sillin L, Vitale G, Xenos ES, Eisen GM, Dominitz J, Faigel D, Goldstein J, Kalloo A, Peterson B, Raddawi H, Ryan M, Vargo J, Young H, Simmang C, Hyman N, Eisenstat T, Anthony T, Cataldo P, Church J, Denstman F, Glennon E, McConnell J, Nogueras J, Orsay C, Otchy D, Place R, Rakinic J, Savoca P, Tjandra J. Principles of privileging and credentialing for endoscopy and colonoscopy. Gastrointest Endosc 2002;55:145-8.

Recebido em 7/4/2004 Aprovado em 30/7/2004 